Revue d'histoire de l'enfance « irrégulière »

Le Temps de l'histoire

18 | 2016

Le psychiatre, l'enfant et l'État

\title{
La guidance du docteur Healy : modulation de la psychiatrie infantile?
}

Doctor Healy's guidance: modulation of child psychiatry?

Guillaume Périssol

\section{(2) OpenEdition}

1 Journals

Édition électronique

URL : https://journals.openedition.org/rhei/3953

DOI : 10.4000/rhei.3953

ISSN : 1777-540X

Éditeur

Presses universitaires de Rennes

Édition imprimée

Date de publication : 30 novembre 2016

Pagination : 213-230

ISBN : 978-2-7535-5175-6

ISSN : 1287-2431

Référence électronique

Guillaume Périssol, « La guidance du docteur Healy : modulation de la psychiatrie infantile? », Revue d'histoire de l'enfance « irrégulière » [En ligne], 18 | 2016, mis en ligne le 30 novembre 2018, consulté le 21 septembre 2021. URL : http://journals.openedition.org/rhei/3953 ; DOI : https://doi.org/10.4000/ rhei.3953 


\section{La guidance du docteur Healy: modulation de la psychiatrie infantile?}

William Healy (1869-1963) a mené une longue carrière de médecin, d'universitaire et de réformateur. Ses idées et ses pratiques en matière de psychiatrie infantile et de traitement de la délinquance juvénile ont profondément marqué, aux niveaux américain et international, le domaine de l'« enfance inadaptée », concept qu'il participe à inventer - étendant ainsi le champ d'intervention de sa spécialité au-delà du noyau dur de la maladie. En 1937, lors du premier congrès international de Psychiatrie infantile, il déçoit en partie les attentes des organisateurs qui proposaient " de montrer l'importance de la "débilité mentale dans la production de la délinquance infantile et juvénile" ", puisqu'il affirme qu'« il n'y a pas de relation causale directe et immédiate entre la déficience mentale et la délinquance ". L'inventeur de la première clinique de guidance infantile se refuse à toute explication monocausale par trop simpliste, insistant sur une conception multifactorielle de la délinquance, ouverte à la psychologie, à la psychanalyse et aux apports du travail social. Sa méthode fait la part de plus en plus belle à l'échange et au dialogue, sur une base psychothérapeutique. Dans le champ psychiatrique, voire au-delà, elle participe d'une nouvelle logique, que l'on reliera au concept de «modulation » proposé par Gilles Deleuze dans son analyse des sociétés de contrôle.

William Healy (1869-1963) had a long career as a doctor, an academic and a reformer. His practice and his ideas on child psychiatry and the treatment of juvenile delinquency left a profound mark on the field of "maladjusted children" in the United States and internationally. He participated in the invention of the concept of maladjustment, thus widening the scope of psychiatry beyond the disease model. At the First International Conference on Child Psychiatry held in 1937, the organizers of the event, who proposed "to show the importance of 'mental deficiency in the development of juvenile delinquency"' were partly disappointed, since Healy claimed that "there is no immediate and direct causal relationship between deficiency and delinquency". The inventor of the first child guidance clinic refused overly simplistic one-cause explanations and he underlined a multi-factorial approach of delinquency which took into account the views of psychology, psychoanalysis and social work. Healy's method was increasingly based on psychotherapy and emphasized dialog and exchange between subjects. In the psychiatric field and beyond this method was part of a new rationale that could be linked to the concept of "modulation" proposed by Gilles Deleuze when he analyzed the societies of control.

\author{
Guillaume PÉRISsOL \\ Chercheur en histoire \\ à l'École nationale de \\ protection judiciaire de \\ la jeunesse.
}


1. SnOdGRASS Jon, « William Healy (1869-1963): Pioneer Child Psychiatrist and Criminologist », Journal of the history of the Behavioral Sciences, vol. 20, Oct. 1984,

p. 332-339.
Mots-clés: William Healy, guidance infantile, délinquance juvénile, psychiatrie Keywords : William Healy, child guidance, juvenile delinquency, psychiatry

Té en 1869 et mort en 1963, le docteur William Healy a mené une longue 1 carrière de psychiatre, d'universitaire et de réformateur, marquant sa discipline de ses idées et méthodes. Né en Angleterre, d'où il émigre à neuf ans pour les États-Unis, il doit, par manque de moyens, arrêter ses études pour exercer à quatorze ans l'emploi de garçon de bureau dans une banque. Grimpant dans la hiérarchie de l'établissement, il se décide pourtant à changer radicalement d'orientation professionnelle. Grâce à l'appui de membres de sa communauté à Chicago, il rentre à l'université Harvard en 1892. De ce parcours, et de l'enseignement qu'il reçoit, avant de faire sa médecine, de William James - un enseignement marqué par la critique de l'intellectualisme et une conception scientifique de la psychologie s'appuyant sur l'observation -, il gardera une vision empiriste et pragmatique du monde, ouvert aux différentes approches, sans jamais vouloir s'enfermer dans une école - ainsi de l'école psychanalytique qu'il découvre en 1906-1907 lors d'un voyage d'étude en Europe ${ }^{1}$.

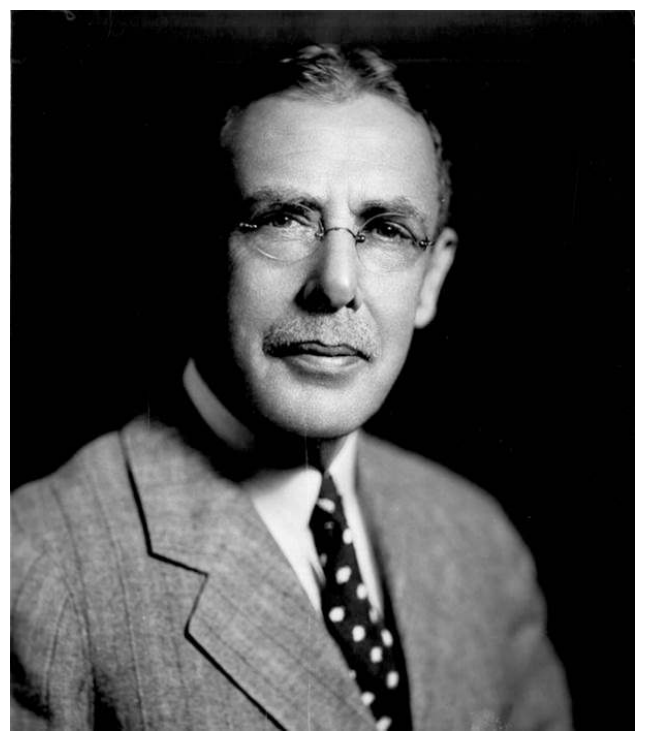

Illustration 1. Docteur William Healy (1869-1963)

Source: Photographie sans date. Copyright (@ 2016 Judge Baker Children's Center. Used with permission. 
Revenu à Chicago en 1907, où il ouvre un cabinet de neurologie, il s'implique très vite dans les réseaux du centre social de Hull House et du tribunal pour enfants de la ville. Cette juvenile court constitue une première mondiale, répondant aux risques de chaos social dans un monde urbain qui explose, et où l'exploitation capitaliste produit fortunes rapides et misère, tensions et violences. Le système de la justice des mineurs (juvenile justice system) se constitue et se perfectionne avec ses disciplines et ses services spécialisés, depuis les services sociaux jusqu'aux institutions de rééducation et de soin. En s'appuyant sur les techniques et savoirs des sciences sociales, psychologiques et médicales, le dispositif vise à connaître les délinquants pour mieux les réintégrer dans la société par une normalisation douce, en évitant si possible la punition de l'enfermement. Avec ces objectifs en tête, et pour répondre aux besoins de la nouvelle juridiction pour mineurs, le docteur Healy crée en 1909 à Chicago le Juvenile Psychopathic Institute, dont l'intitulé traduit encore l'approche pathologisante du phénomène de la délinquance juvénile. En 1917, il quitte Chicago pour prendre la direction de la Judge Baker Foundation, renommée en 1933 Judge Baker Guidance Center. Le succès des cliniques de guidance infantile est national et international, parallèle à celui des tribunaux pour enfants, dans la première moitié du $\mathrm{xx}^{\mathrm{e}}$ siècle. Rien qu'aux États-Unis, on compte plus de quarante clinics en $1933^{2}$. Concernant l'Europe, les travaux de John Stewart ont mis en lumière l'importance du mouvement en Grande-Bretagne, soulignant également ses limites, ses travers et ses impasses ${ }^{3}$.

Recevant de son vivant le titre de "père de la guidance infantile ", Healy fonde sa pratique sur une conception multifactorielle de la délinquance, refusant l'explication monocausale par l'hérédité, ce qui l'amène à s'opposer aux thèses de Cesare Lombroso, qu'il juge peu scientifiques. L'individu délinquant (The Individual Delinquant), qu'il publie en 1915, est une réponse critique à L'homme criminel (L'uomo delinquente) ${ }^{4}$. Arthur E. Fink, dans une célèbre étude sur les causes du crime ${ }^{5}$, considérera qu'Healy ouvre ainsi une nouvelle ère dans la criminologie. Le caractère novateur de ses recherches, souvent menées conjointement à partir de 1913 avec sa future femme, la psychologue Augusta Bronner, doit cependant être replacé dans le contexte des États-Unis de la Progressive Era. L'optique réformatrice des child savers (sauveurs d'enfants) peut paraître a posteriori bien conservatrice, utile au contrôle des classes populaires par les classes dirigeantes qui s'achèteraient ainsi une bonne conscience ${ }^{6}$. De manière générale,
2. SCHNeIDER Eric C., In the Web of Class: Delinquents and Reformers in Boston, 1810s-1930s, New York, New York University Press, 1992, p. 172.

3. Stewart John, Child Guidance in Britain, 19181955: The Dangerous Age of Childhood, London, Pickering \& Chatto, 2013.

4. Lettre de William Healy à Julia Lathrop, du 4 avril 1908, citée dans: TANENHAUS David S., Juvenile Justice in the Making, New York, Oxford University Press, 2004, p. 117.

5. Fink Arthur E., Causes of Crime, New York, Barnes, 1938.

6. L'ouvrage pionnier d'Anthony M. Platt sur « l'invention » de la délinquance juvénile critique dès 1969 le soi-disant progressisme américain du tournant des $\mathrm{XIX}^{\mathrm{e}}$ et $\mathrm{XX}$ e siècles. PLATT Anthony M., The Child Savers: The Invention of Delinquency, expanded $40^{\text {th }}$ anniversary edition, New Brunswick, New Jersey, Rutgers University Press, 2009. 
7. Getis Victoria, The Juvenile Court and the Progressives, Urbana, University of Illinois Press,

2000.

8. Le Commonwealth Fund est qualifié de " conservative innovator » dans Horn Margo, Before It's Too Late: The Child Guidance Movement in the United States, 1922-1945, Philadelphia, Temple University Press, 1989,

9. Burt Cyril, The Young Delinquent, New York, Appleton, 1925.

10. JONES Kathleen W. " "Mother Made Me Do It": Mother-Blaming and the Women of Child Guidance ", LADD-TAYLOR Molly and UMANSKY Lauri (eds.), Bad Mothers: the Politics of Blame in 20th Century America, New York, New York University Press, 1998, p. $99-100$ les sciences du psychisme et les autres sciences intervenant dans le traitement de la délinquance juvénile vont trouver là un terrain d'expérimentation et de légitimation, constituant un savoir et un pouvoir, dont la fonction régulatrice semble assurer avant tout le maintien de l'ordre en place - ce qui explique sans doute la prospérité de ces approches scientifiques et techniques, comme la guidance infantile, qui, ciblant des cas particuliers, renoncent à voir, sinon à régler, les problèmes structurels, sociaux et économiques ${ }^{7}$. Le financement des cliniques de guidance par le Commonwealth Fund, une fondation initiée par la veuve d'un associé de John D. Rockefeller, tout à la fois « innovatrice " et " conservatrice ${ }^{8}$ ", paraît soutenir cette hypothèse. Healy sera d'ailleurs critiqué pour son manque d'attention à la pauvreté ${ }^{9}$ et aux réalités sociologiques que sa méthode centrée sur l'individu prend finalement peu en compte.

Dans son champ disciplinaire, Healy participe d'un renouvellement profond de la psychiatrie infantile, qui, emboîtant le pas à une justice des mineurs éducative et anti-carcérale, se réinvente hors des murs de l'internement. S'il laisse quelques patients irrécupérables à l'asile, il accueille dans sa clinique toutes sortes d'enfants à problème, devenus plus nombreux que les jeunes délinquants, souvent d'origine populaire, qu'il prétendait initialement soigner, comme une manière de prévenir les tendances antisociales quelles qu'elles soient. Dès le milieu des années 1930, la moitié des patients seulement sont encore adressés au Judge Baker Guidance Center par la justice ou les services sociaux ${ }^{10}$, marquant de fait le passage à une nouvelle logique de contrôle, plus douce et plus étendue, assurant la collaboration de familles de la middle-class, qui, inquiètes des anormalités et bizarreries de leur enfant, se présentent de leur propre initiative aux portes de la clinique.

C'est donc en rompant avec les vues traditionnelles de l'aliénisme et de la psychiatrie du XIX ${ }^{e}$ siècle, comme le prouve son exposé au congrès international de Psychiatrie infantile de 1937, que le docteur Healy tend à déplacer l'axe de sa discipline du disease au disorder, de la maladie au trouble. Son système d'analyse est éclectique, ouvert à la psychologie, à la psychanalyse et aux apports du travail social. Il trouve peut-être une unité dans la notion de comportement, pensé comme le produit de la personnalité, de la psyché et de la vie affective, sous influence de l'environnement - le problème des constitutions et du biologique passant à l'arrière-plan. Sa méthode de guidance paraît répondre aux besoins d'une juvenile court centrée sur l'individu, dont l'action régulatrice supplée aux 
modes habituels de punition et de surveillance de plus en plus contestés. Faisant la part belle à l'échange et au dialogue, sur une base psychothérapeutique, elle semble correspondre aux «modulations » que Gilles Deleuze définit dans son analyse des sociétés de contrôle. Selon le philosophe, les sociétés de contrôle auraient succédé aux sociétés disciplinaires, caractérisées par l'enfermement, le «moule ». En lieu et place de la prison et de tous les milieux clos qui s'en inspirent et qui rentrent en crise au $\mathrm{xx}^{\mathrm{e}}$ siècle (l'usine, l'école, etc.), ne voit-on pas surgir le "nouveau monstre " du contrôle, avec sa logique de modulation, parfaitement individualisée et évolutive dans l'interaction, " comme un moulage auto-déformant qui changerait continûment, d'un instant à l'autre ${ }^{11}$ "? La guidance du docteur Healy serait-elle à interpréter comme une modulation de la psychiatrie infantile, sortie du moule asilaire, pour reprendre la métaphore deleuzienne?

\section{GUIDANCE INFANTILE ET PSYCHIATRIE JURIDIQUE}

En 1937, lors du premier congrès international de Psychiatrie infantile, Healy revient aux sources de sa carrière de psychiatre, puisqu'il intervient dans la section de psychiatrie juridique. Sans doute a-t-il dû surprendre, décevoir ou agacer les organisateurs qui proposaient « de montrer l'importance de la "débilité mentale dans la production de la délinquance infantile et juvénile"12 ». Il se méfie en effet de tels liens de causalité - la liaison trop rapide entre débilité et délinquance risquant d'aboutir à la marginalisation du déviant, voire à sa relégation dans une sorte de sous-humanité, au destin carcéral ou asilaire, pour laquelle il n'y a que peu d'espoir. La position progressiste d'Healy (progressiste au sens où elle vise à intégrer et non à exclure, sans préjuger des éventuels effets pervers et normalisateurs de l'intégration elle-même) s'affirme de plus en plus nettement au cours de ses recherches, dont les conclusions rejoignent celles d'autres grandes enquêtes américaines ${ }^{13}$, comme l'enquête de Carl Murchison. Ce dernier avait abouti en 1926 à des résultats "spectaculaires ", obtenus à partir d'un vaste échantillon de plusieurs milliers de prisonniers, qu'il estimait in fine n'être pas intellectuellement différents de la population dite "normale ${ }^{14}$ ». Dans une lettre " un peu ironique et sceptique » au médecin français Georges Heuyer, qui préparait le congrès et l'interrogeait sur cette thématique, Healy écrivait donc « qu'il ne comprenait pas beaucoup l'intérêt de cette question et que tout avait été dit à ce sujet ${ }^{15}$ ». Le jour de son intervention à Paris, il affirme encore:
11. Deleuze Gilles, «Postscriptum sur les sociétés de contrôle ", texte de mai 1990 in Pourparlers, 1972-1990, Paris, Les Éditions de Minuit, 2005, p. 241-242.

12. Programme du premier congrès international de Psychiatrie infantile, 1937 (Archives Georges Heuyer, université Paris 8).

13. Sauf précision, le mot " américain " sera employé relativement aux États-Unis d'Amérique.

14. Nony Camille, «Délinquance et infériorité de l'intelligence ", L'Année psychologique, t. 51, 1949, p. 36.

15. HeuYer Georges, "Discours de M. le docteur Heuyer, Président du Congrès ", discours de clôture in Comptes rendus, par Maurice Leconte, du premier Congrès international de psychiatrie infantile, Lille, SILIC, 1937, p. 231. 
16. Healy William, «The Relationship of Mental Deficiency to Delinquency ", deuxième rapport in III. Rapports de psychiatrie juridique du premier Congrès international de psychiatrie infantile, Lille, SILIC, 1937, p. $24-25$ et p. 30 Sauf mention contraire, les traductions des archives et sources en langue anglaise sont fournies par l'auteur.

17. Ainsi le docteur Wintsch de Lausanne, qui semble ne rien avoir entendu ou ne rien vouloir entendre de l'exposé de Healy et du débat qu'il a provoqué, puisqu'il déclare:

«On a longtemps considéré les enfants délinquants comme des dévoyés. On admet maintenant que ce sont souvent des débiles mentaux » («Discussion des rapports de psychiatrie juridique ", Comptes rendus..., op. cit., p. 187)

18. Nony Camille, «Délinquance... », op. cit. p. 37.

\begin{abstract}
Il est donc impossible d'expliquer la délin$\ll_{\text {quance en termes de quotient intellectuel. En }}$ d'autres mots, il n'y a pas de relation causale directe et immédiate entre la déficience mentale et la délinquance. [...] La délinquance des déficients mentaux a dans la plupart des cas exactement la même cause que la délinquance en général. Elle est principalement le résultat des circonstances sociales, des pressions sociales (avec tout ce que ce terme implique du point de vue sociologique), d'une éducation défaillante et du manque d'occasions pour que les idéaux du moi [ego-ideals] se forment correctement ${ }^{16}$.
\end{abstract}

Healy est dans la lignée de ses travaux les plus récents, et en particulier de New Light on Delinquency and Its Treatment, publié l'année précédente avec sa femme, psychologue et spécialiste de l'intelligence des enfants, qui défendit très tôt, dès son doctorat publié en 1914, l'idée que la grande majorité des jeunes délinquants possédaient une intelligence normale. Si la thèse de l'arriération mentale, qui était largement admise avant-guerre, est fermement rejetée par le psychiatre américain, elle garde encore des défenseurs, comme le prouvent les discussions lors du congrès ${ }^{17}$, même si, dans les années 1930, la tendance est à considérer que la déficience mentale (dont la définition et la mesure font néanmoins toujours débat) ne touche qu'un pourcentage réduit de délinquants: de 8 à $13 \%$, alors que les auteurs de 1910-1914 aboutissaient en moyenne à un résultat de $50 \%$, selon les estimations de Camille Nony pour L'Année psychologique ${ }^{18}$. Certains comptes rendus de l'évènement parisien publiés dans les revues 
spécialisées ne cachent pas l'effet qu'a pu produire l'intervention de Healy. En Belgique, où le directeur du Judge Baker Guidance Center est régulièrement cité comme un " grand savant », voire «l'une des premières autorités en matière de délinquance juvénile ${ }^{19}$ ", le professeur Paul Vervaeck note diplomatiquement que son " riche " exposé " amena peut-être quelque désenchantement " et relativise les conclusions de l' "éminent spécialiste » dans la Revue de droit pénal et de criminologie ${ }^{20}$.

À la fin d'une intervention où il « dépathologise " largement la délinquance juvénile pour mieux l'intégrer dans la problématique plus vaste de l'inadaptation, il conclut que la question du discernement du mineur, que les juges français posent depuis 1810 (en application de l'article 66 du Code pénal), et qui intéresse au plus haut point leurs experts psychiatres $^{21}$, n'a tout simplement pas de sens. Elle relève de la métaphysique, non de la compétence du juge pour enfants, qui exerce son autorité en bon père de famille ${ }^{22}$. Le point de vue de Healy s'accorde une fois de plus au point de vue général des psychiatres américains les plus en pointe, qui se battent, depuis le début du $\mathrm{xx}^{\mathrm{e}}$ siècle, pour que soit mis en œuvre un examen psychique complet de l'accusé, qui dépasserait la question de savoir s'il était ou non dans un état de folie l'empêchant de distinguer le bien du mal. Plus spécifiquement, à partir de sa position de spécialiste de l'enfance et de l'adolescence, ses théories et pratiques consistent objectivement à déplacer le centre de gravité du champ psychiatrique de la maladie au trouble, du disease au disorder, l'étude et le traitement des jeunes délinquants servant de levier à une telle entreprise.

\section{DISEASE ET DISORDER}

Refusant toute théorisation excessive, Healy suit et influence à la fois la perspective des juges pour enfants et des travailleurs sociaux qui les entourent, concentrant ses analyses sur l'individu qu'il faut réhabiliter, plus que sur le groupe auquel il appartient ou les conditions socio-économiques difficiles qu'il pourrait subir. Dans la lignée d'un Granville Stanley Hall qui rappelait la nécessité dans ses travaux sur l'adolescence d'étudier scientifiquement, et un par un, les jeunes délinquants, il ne cesse d'insister dans sa spécialité sur le cas comme espace de connaissance et moyen d'intervention - les troubles mentaux des hommes revenus de la première guerre mondiale, qui ne connaissaient pas précédemment de problème particulier de comportement, ayant pu

\author{
19. RACINE Aimée, \\ "L'évolution des idées aux \\ États-Unis en matière de \\ délinquance juvénile ", Le \\ service social, nos $4-5$, avril- \\ mai 1931, p. 52. \\ 20. Vervaeck Paul, « Le rôle \\ criminogène de la débilité \\ mentale étudié au Congrès \\ de psychiatrie infantile", \\ Revue de droit pénal et \\ de criminologie, 1937 , \\ p. 932-938. Tous mes remer- \\ ciements à Veerle Massin \\ pour les références et pour \\ les informations données \\ sur la réception de Healy en \\ Belgique. \\ 21. Programme du premier \\ Congrès international de \\ psychiatrie infantile, 1937 \\ (Archives Georges Heuyer, \\ université Paris 8). \\ 22. Healy William, «The \\ Relationship... ", op. cit. \\ p. 28-29.
}


encore accentuer et légitimer ce processus d'individualisation du phénomène. En faisant du cas par cas, escamotant de la sorte les enjeux proprement politiques de la délinquance et des questions qu'elle soulève, Healy participe à renforcer les orientations conservatrices de la juvenile court et du social work au détriment des tentatives de transformation et de critique sociales qui avaient pu initialement les animer.

En fin de carrière, au milieu des années 1930, il livre avec Augusta Bronner les résultats d'une de leurs dernières enquêtes, menée pour l'Institut des relations humaines (Institute of Human Relations) de l'université Yale. Un schéma synthétise leur approche, qui se veut complète, ne rejetant a priori aucun paramètre.

THE GENERAL LIFE STREAM OF FEELINGS AND ACTIVITIES

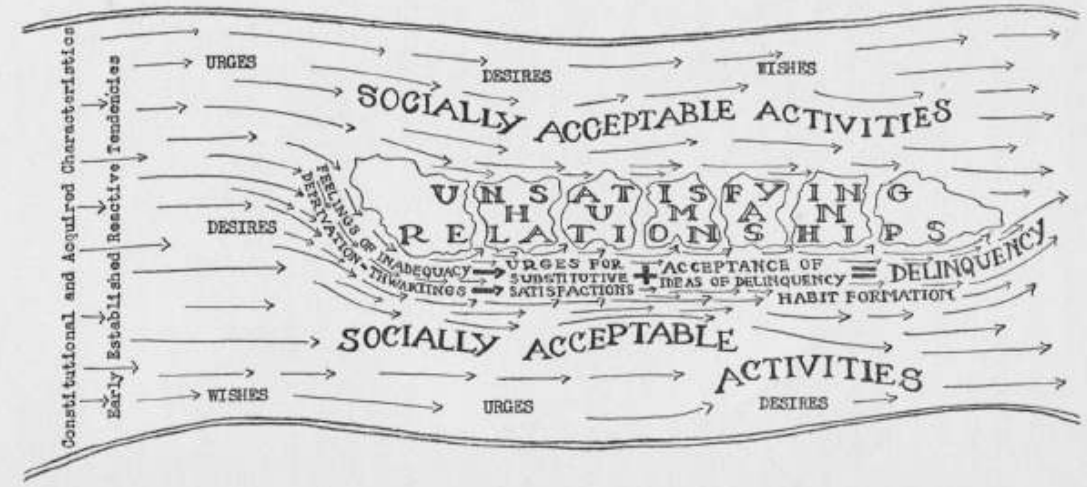

Illustration 2. "Diagram Showing the Development of Delinquent Behavior" (Schéma montrant le développement du comportement delinquant) par le docteur William Healy et Augusta F. Bronner (1936)

Source: Healy William and Bronner Augusta F., New light on Delinquency and Its Treatment: Results of a Research Conducted for the Institute of Human Relations, Yale University, $1^{\text {st }}$ edition 1936, New Haven, published for the Institute of Human Relations by Yale University Press, 1938, p. 4.

L'hérédité est ainsi reconnue comme la source d'un certain nombre de " tendances », mais sa position en amont reste périphérique dans cette représentation du comportement délinquant pensée à partir des interventions possibles, imaginables et souhaitables des sciences du psychisme. La psycho-dynamique est mise en valeur, symbolisée par les flèches, qui sont autant de flux alimentés par les pulsions, les désirs et les envies. Dans le cadre de cette conception du 
sujet, qui vise moins à canaliser qu'à guider et orienter en souplesse les « sentiments " et les " activités ", trois séries principales d'éléments déterminent l'état de santé mentale ${ }^{23}$ : les facteurs externes, auxquels on s'adapte plus ou moins; les relations interindividuelles et affectives, qui permettent au psychiatre ou au psychologue d'agir sans toucher aux conditions structurelles de la vie du sujet (c'est-à-dire l'organisation sociale, économique...); et surtout les conflits internes, le travail de la "santé mentale " consistant, selon Claude-Olivier Doron, « à traduire des conflits réels, mettant en jeu les conditions d'existence au niveau économique et social, en conflits internes à l'individu, qui peuvent et doivent être résolus harmonieusement au niveau de l'individu, sans intervenir sur lesdites conditions ${ }^{24}$ ».

Le contrôle, visant à éviter ou à contourner les « relations humaines insatisfaisantes ", explicitement reliées à la délinquance, semble donc avoir pris la place, auparavant primordiale, du soin, qui avait en objectif la guérison - le terme de control revenant d'ailleurs à de nombreuses reprises dans l'étude de Healy et Bronner, censée apporter un «nouvel éclairage sur la délinquance et son traitement ${ }^{25}$ ». C'est que le directeur du Judge Baker Guidance Center cherche d'abord à résoudre les comportements asociaux. N'a-t-il pas œuvré en 1924 à la fondation de l'Association américaine d'orthopsychiatrie (ou psychiatrie du maintien "droit») ? Cette association, qui a initialement eu pour but d'apporter " un point de vue médical ou neuropsychiatrique sur la délinquance ${ }^{26}$ ", a d'ailleurs fini par s'éloigner du discours criminologique pour s'intéresser aux problèmes du comportement humain en général et corriger le plus tôt possible les déviances, dans l'esprit de la guidance infantile. Comme le révèle le schéma tiré de l'ouvrage de Healy et Bronner, la correction des déviances ne passe pas par un processus de classification puis de normalisation simple, déplaçant l'anormal d'une case à l'autre des nomenclatures médicales en espérant l'en faire sortir, elle fonctionne sur un mode plus subtile, complexe et flexible. La ligne droite se fait courbe, le droit chemin ondoie et autorise certaines voies de traverse. Les flèches ne pointent plus un point précis, mais ondulent dans l'espace.

Plus que sur l'opposition du sain et du pathologique, Healy joue sur les gradations du normal à l'anormal, produisant des courants pouvant porter le jeune vers l'écueil de la délinquance. Vision angoissante d'un monde en train de changer rapidement, gagné par la culture de masse et la remise en cause de

\author{
23. Je reprends ici l'analyse \\ de Doron Claude-Olivier, \\ "L'émergence du concept \\ de " santé mentale » dans \\ les années 1940-1960: \\ genèse d'une psycho-poli- \\ tique ", Pratiques en santé \\ mentale, $n^{\circ} 1,2015$, p. 3-16. \\ 24. Ibid., p. 10. \\ 25. HealY William and \\ Bronner Augusta F., New \\ Light on Delinquency and \\ Its Treatment: Results of a \\ Research Conducted for the \\ Institute of Human Relations, \\ Yale University, $1^{\text {st }}$ edition \\ 1936, New Haven, Published \\ for the Institute of Human \\ Relations by Yale University \\ Press, 1938.
}

26. Cité dans WAGNER Grant H., William Healy, M. D. : Father of the American Child Guidance Movement, Master of Arts, University of Maryland, 1981, p. 55. 
27. Voir AREndt Hannah, La crise de la culture [Between

Past and Future, $1^{\text {re }}$ éd 1961], Paris, Gallimard, 1995.

28. Stewart John, «The Scientific Claims of British Child Guidance, 19181945 », The British Journal for the History of Science, vol. 42, n³, Sep. 2009 p. 429 et suiv.

29. GOKAy Fahreddin Kerim "Les délits infantiles en Turquie ", huitième rapport in III. Rapports..., p. 121

30. Lefaucheur Nadine,

«Psychiatrie infantile et délinquance juvénile: Georges Heuyer et la question de la genèse

« familiale » de la délinquance ", MucCHIELLI Laurent (dir.), Histoire de la criminologie française, Paris, L'Harmattan, 1994. p. 313-332

31. Voir CoREA Joseph Carlo, Racial Delinquency: ItalianAmerican and AfricanAmerican Adolescent Identity and the Delinquency Experience, 1915-1932,

Ph. D. thesis, History, State University of New York at Stony Brook, May 2001. l'autorité, dans et hors de la famille ${ }^{27}$. Comment élever ses enfants, leur éviter la délinquance en pleine crise des années trente? Alors que les totalitarismes tournent le dos à la raison, les découvertes scientifiques ne sont pas rassurantes ${ }^{28}$. Heisenberg n'a-t-il pas énoncé, il y a peu, son troublant principe d'incertitude? La conception des déviances selon Healy repose sur une morale qui n'est guère interrogée, mais a l'avantage d'apporter une certaine stabilité à un univers qui en manque et semble entrer dans une crise majeure, tant économique et sociale que politique et culturelle. Une approche éclectique et souple fournit cette sorte de stabilité que la trop grande rigidité de l'épistémè précédente n'assurait plus.

\section{ÉCLECTISME THÉORIQUE ET TRAVAIL D'ÉQUIPE}

Le télescopage de différentes théories n'a rien d'exceptionnel dans la pensée psychiatrique de l'époque, comme en témoignent plusieurs rapports parvenus au congrès de 1937, qui insistent parallèlement sur la multiplicité des facteurs à prendre en compte pour expliquer le comportement d'un sujet. Par exemple, le professeur Gokay de Turquie rappelle avec bon sens que la misère entraîne la «chute " de nombreux " malheureux ${ }^{29}$ " - fatalité avant tout sociale, que l'on observe également dans les pays les plus riches en ces temps de Grande Dépression. Aussi les contorsions discursives de certains spécialistes du psychisme pour que l'édifice conceptuel qu'ils bâtissent tienne debout, et résiste à l'épreuve des faits et à l'usure des théories, ont suscité bien des critiques, ainsi de Heuyer au sujet des rapports entre le milieu et l'héréditée ${ }^{30}$. Loriginalité d'Healy réside dans un refus assez net du déterminisme et des typologies effrayantes qui en ressortent, tel le criminel-né de Cesare Lombroso ou le pervers constitutionnel d'Ernest Dupré. Sa pratique est certes plus ambiguë, en particulier quand il a affaire à des jeunes immigrés ou à des jeunes Noirs, la logique WASP de son raisonnement pouvant prendre le dessus et générer des discriminations raciales ${ }^{31}$. Probablement, la norme américaine du «Blanc, anglo-saxon et protestant " est d'autant plus prégnante à Boston que l'on se trouve en Nouvelle-Angleterre.

Le docteur Healy est un promoteur important des rencontres disciplinaires: avec la psychologie bien sûr, mais aussi avec la psychanalyse, qu'il participe à introduire aux États-Unis. Il suivra d'ailleurs une courte analyse didactique avec Franz Alexander, venu de Berlin pour occuper à Chicago la première chaire universitaire de psychanalyse. Mais jugeant la psychanalyse trop intuitive et 
insuffisamment scientifique, il ne la pratiquera lui-même jamais ${ }^{32}$. En fonction d'une conception relativement sage et institutionnalisée de cette nouvelle méthode thérapeutique, il en propose une «forme modifiée $e^{33}$ " visant le social adjustement au monde moderne. Une telle approche normalisatrice, objet de polémiques dans l'histoire du mouvement analytique international, s'expliquerait entre autres par le conformisme dominant la société américaine, les Européens ayant beau jeu de railler les Américains sur ce thème (sans forcément en explorer les tenants et aboutissants). «La psychanalyse leur va comme une chemise blanche à un corbeau ${ }^{34}$ ", ironisait Freud. Ainsi est-il peut-être possible de comprendre la longévité dans son pays, jusqu'après la seconde guerre mondiale, des travaux " approfondis " et " scientifiquement étayés " d'Healy, dont le sérieux et la pertinence sont évalués à l'aune de leur utilité concrète et de leur efficacité pressentie dans le contexte américain. Certaines approches jugées trop « provocantes » de la psychanalyse seront, elles, délaissées ${ }^{35}$.

Se détachant de la base neurologique de sa discipline, le docteur Healy met de plus en plus l'accent sur les rapports affectifs et éducatifs dans le traitement des inadaptés, favorisant le maintien dans la famille, avec si nécessaire une psychothérapie de l'enfant ou des parents. La prise en charge est assurée à partir d'une série d'éléments, dont on retrouve une liste dans L'individu délinquant de $1915^{36}$ : l'histoire familiale, le développement de l'enfant depuis sa naissance et pendant la grossesse, l'environnement dans lequel il vit, son évolution psychique et morale, ainsi que le compte-rendu des actes de délinquance. Pour établir diagnostic et pronostic, des examens médicaux et psychologiques complètent les données recueillies auprès des parents, des enseignants, des voisins, des forces de l'ordre... et de l'enfant lui-même, le tout étant conservé dans un dossier complété au fur et à mesure du suivi, grossissant en fonction de la complexité et de la durée du traitement.

À la clinique de guidance infantile de Boston, le travail se fait en équipe avec les psychologues et les travailleurs sociaux, dans une ambiance qui se veut détendue et amicale. La manière dont se donne à voir Augusta Bronner, la main posée sur l'épaule de l'enfant qu' elle teste (ill. 3), est emblématique d'un nouveau rapport au délinquant, fait d'attention, de bienveillance et de confiance, qui subsume le champ de la psychiatrie infantile puisqu'on le retrouve dans les représentations des relations du mineur avec son juge ou avec son agent de probation $^{37}$. Bien loin de la prison ou de l'asile psychiatrique, en somme.
32. WAGner Grant H., William Healy..., op. cit., p. 61.

33. E. W. Burgess cité dans: GITRE Edward J. K. «Importing Freud: FirstWave Psychoanalysis, Interwar Social Sciences, and the Interdisciplinary Foundations of an American Social Theory ", Journal of the History of the Behavioral Sciences, vol. $46, n^{\circ} 3$, été 2010, p. 245.

34. Michaud Stéphane (éd.), Correspondances de Freud, Paris, Presses Sorbonne Nouvelle, 2007, p. 63.

35. Brown James IV, « Child Welfare Classics ", Social Service Review, vol. 34, n² June 1960, p. 195-202.

36. Liste reprise dans Getis Victoria, The Juvenile Court..., op. cit., p. 73.

37. PérISSOL Guillaume, " "The Quality of Mercy is not Strain'd": Ideological and Repressive Modes of Juvenile Justice - A Comparison between Paris and Boston in the Mid-Twentieth Century ", Journal of Social History, vol. $48, n^{\circ} 2$, Winter 2014 p. 295 et suiv. URL: http:// jsh.oxfordjournals.org/ content/48/2/289. 
38. ThIERCÉ Agnès, Histoire de l'adolescence, 18501914, Paris, Belin, 1999. La périodisation américaine est assez proche de celle tracée par Agnès Thiercé pour la France.

39. Pour reprendre quelques termes employés à l'occasion d'un procès intenté, en 1907, par une chambre de commerce «forcée » de déménager suite à l'arrivée dans l'immeuble du Tribunal pour enfants de Chicago, avec ses populations « indésirables », souvent immigrées (cas cité dans: Tanenhaus David S., Juvenile Justice..., p. 27-28).

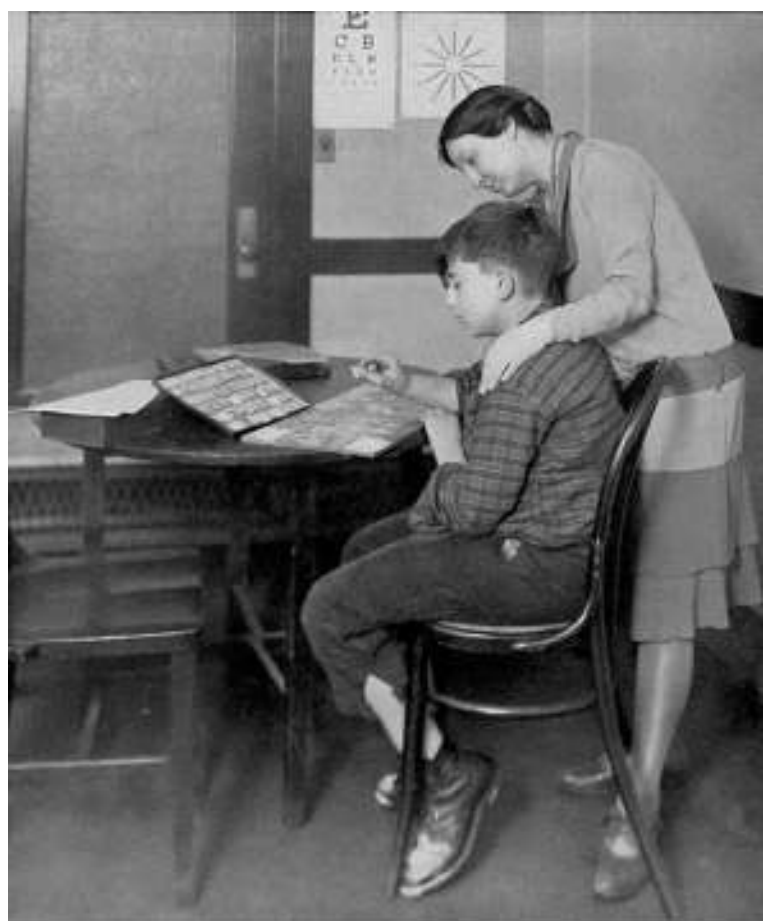

Illustration 3. Augusta F. Bronner testant un enfant à la Judge Baker Foundation de Boston. Source: Photographie sans date (entre 1917 et 1932, quand la fondation était localisée au 40 court Street, à Boston). Copyright (c) 2016 Judge Baker Children's Center. Used with permission.

Il faut voir dans ce geste plus qu’une donnée publicitaire, liée à la nécessité de vendre les juvenile courts ou leurs institutions auprès du public, et considérer les acteurs dans leur sincérité même, dont la signification les dépasse, car elle s'inscrit dans l'évolution des mours et du ressenti social. Le sentiment de l'enfance, puis de l'adolescence ${ }^{38}$, encourage la reconnaissance d'une commune humanité. Cette manière d'outrepasser les frontières de classe, alors que le jeune délinquant reste parfois décrit, en particulier avant la Grande Guerre, comme un être misérable, sale et malodorant, voire "vicieux » et "dépravé39 ", produit des classes laborieuses assimilées aux classes dangereuses, peut aussi être reliée au long processus de démocratisation analysé par Alexis de Tocqueville. Dans son œuvre, la démocratie est un concept ambivalent, recoupant deux significations principales: un régime politique défini par le gouvernement du peuple par le 
peuple et, surtout, un "état social démocratique » caractérisé par le refus des hiérarchies héritées et une tendance irrésistible à l'égalisation ${ }^{40}$. Ainsi pensé, le développement démocratique apparaît particulièrement précoce aux États-Unis: l'auteur de De la démocratie en Amérique remarquait dès les années 1830 que, dans ce pays, on "n'accorde guère de grands bienfaits", mais on "rend sans cesse de bons offices ${ }^{41}$ ». Si les liens entre les hommes se sont affaiblis, le cercle de ses semblables s'est élargi par le mouvement d'égalisation des conditions et il est de moins en moins possible et imaginable de classer à part ou d'exclure des catégories entière de la population, en particulier les catégories les plus fragiles, comme celles des enfants et des adolescents, qui suscitent de plus en plus d'attention.

Avec en toile de fond ce progrès du sentiment démocratique et du sentiment de l'enfance et de l'adolescence, qui ne manque pas d'affecter le fonctionnement du système pénal, la guidance infantile s'inscrit politiquement dans le courant maternaliste. Les protagonistes de ce mouvement étaient des femmes engagées dans la construction d'un système de protection sociale, plus particulièrement attachées à défendre femmes et enfants en faisant valoir leur rôle de mère sur la scène publique. Du maternalisme qui s'épanouit durant la Progressive Era, la guidance infantile tire certaines caractéristiques, comme la confiance dans la science ou la sensibilité au social, même si le regard se teinte souvent d'un jugement de classe. Mais elle s'en détache par la place centrale qu'elle donne à l'étude et à la prise en charge de l'individu en tant que tel, reléguant finalement à l'arrière-plan les facteurs jugés extérieurs pour se concentrer sur le comportement. Le mouvement de guidance infantile retournera même la problématique maternaliste contre les femmes elles-mêmes lorsqu'elles seront accusées d'être de mauvaises mères et que la guidance infantile se fera familiale dans l'entredeux-guerres ${ }^{42}$, étendant son contrôle.

\section{Modulations DU CONTRÔLE}

Les constructions disciplinaires, modélisées autour du panopticon savamment imaginé par Jeremy Bentham, commencent à se fissurer au début du $\mathrm{xx}^{\mathrm{e}}$ siècle, alors qu'elles paraissaient des plus solides. De nouvelles techniques de contrôle, parfois fort simples, vont rencontrer un énorme succès, comme la probation, réinventée et modernisée au milieu du XIx ${ }^{\mathrm{e}}$ siècle par un obscur cordonnier de Boston, John Augustus. La logique probationnaire de l'échange

\author{
40. LAMBERTI Jean-Claude, \\ Tocqueville et les deux \\ démocraties, Paris, Presses \\ Universitaires de France, \\ 1983. \\ 41. Tocqueville Alexis de, De \\ la démocratie en Amérique, \\ version parue en 1840 , \\ Textes essentiels: anthologie \\ critique, présentée par Jean- \\ Louis Benoît, Paris, Pocket, \\ 2000, p. 107. \\ 42. JoNEs Kathleen W., \\ Taming the Troublesome \\ Child: American Families, \\ Child Guidance, and \\ the Limits of Psychiatric \\ Authority, Cambridge \\ (Mass.) and London \\ (England), Harvard \\ University Press, 1999, \\ p. 175.
}


43. Beers Clifford W., A Mind That Found Itself: An Autobiography, New York, Longmans, Green, and Co.,

1908, p. 1.

44. De ce point de vue, les États-Unis, ou tout au moins certains de ses États, semblent en avance sur la France si l'on en croit les statistiques portant sur les durées de séjour dans les écoles de réforme du Massachusetts - durées bien plus courtes que dans les internats de rééducation français, où la croyance en leur efficacité éducative se maintient plus longtemps (en lien également avec la volonté de fournir un cycle complet de formation, sur plusieurs années).

45. Comme nous avons pu le constater dans les dossiers consultés aux archives du Departement of Youth Services du Massachusetts.

46. Voir, par exemple, la plupart des illustrations utilisées dans: Actes du douzième Congrès pénal et pénitentiaire international, La Haye, 14-19 août 1950, Berne, 1951, Commission internationale pénale et pénitentiaire, vol. 1-vol. 2. et du dialogue, en lieu et place du regard panoptique qui voit sans être vu, va faire fonctionner le système de la justice des mineurs, laboratoire d'expérimentation d'un soft control qui gagnera progressivement l'ensemble de l'appareil judiciaire, et au-delà, atteignant l'appareil psychiatrique. Au sein de ce contexte favorable à certaines formes de compassion et d'écoute, la voix d'un homme comme Clifford Beers peut être entendue. Dans une autobiographie choc, le fondateur du mouvement américain d'hygiène mentale raconte les douloureuses expériences qu'il vécut lorsque son esprit devint la proie d'une "guerre civile ${ }^{43}$ ".

Le livre de Beers sort un an avant l'ouverture par Healy de sa première clinique à Chicago. Dans une société à visée inclusive, l'hygiène mentale, qui deviendra "santé » mentale sous l'influence de professionnels comme Healy, nourrit le secteur de l'inadaptation. Les institutions fermées y semblent de plus en plus réservées aux cas les plus graves ou limitées au traitement ponctuel de courte durée, puisque l'on est de moins en moins convaincu de la vertu réhabilitative et curative sur le long terme de l'enfermement ${ }^{44}$. Elles évoluent d'ailleurs, sinon dans leurs pratiques, du moins dans leur présentation. Il faut noter une fois de plus l'avancée américaine en la matière. La Lyman Training School avait été la première école de réforme publique, pour garçons délinquants, à ouvrir aux États-Unis, à Westborough dans le Massachusetts, en 1846. Un siècle plus tard, dans les années 1940, elle cherche à communiquer une nouvelle image d'elle-même, employant une psychologue qui s'inspire des méthodes de la guidance infantile, et qui note soigneusement tous les problèmes de comportement (masturbation, ongles rongés...) ${ }^{45}$.

La mise en scène du photographe souligne l'importance de cette spécialiste du psychisme, qui paraît présider la réunion de travail décidant de la classification et du placement des enfants (ill. 5).

Tandis que la plupart des institutions de rééducation européennes montrent encore le visage de la discipline, avec des jeunes défilant au pas dans un ordre tout militaire ${ }^{46}$, l'école de réforme de Westborough insiste sur le jeu et le plaisir comme mode de développement et d'épanouissement. 


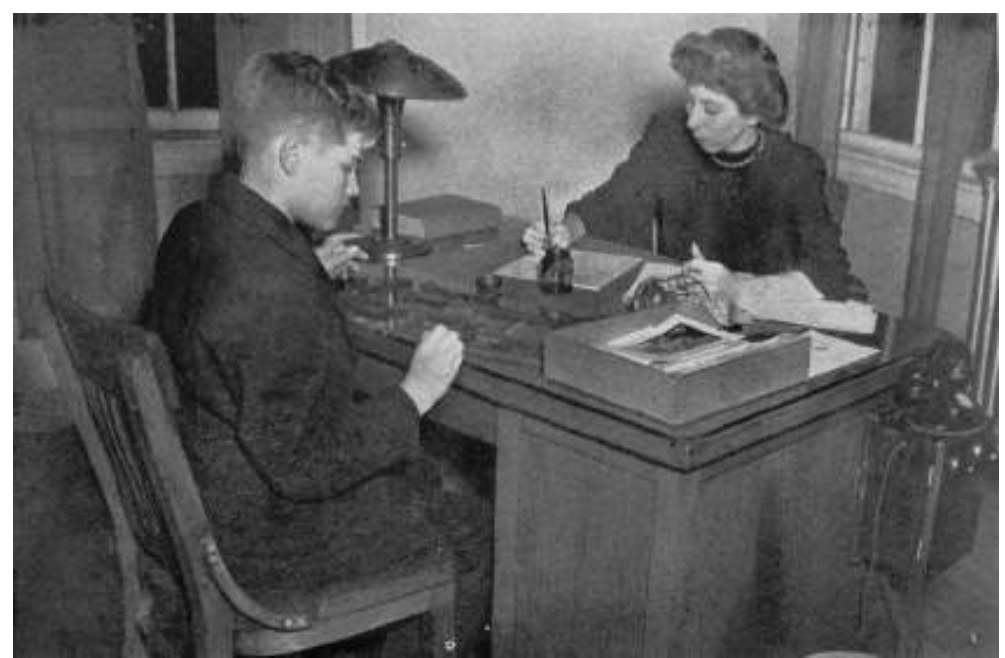

Illustration 4. "Mental measurement" (tests psychologiques) à la Lyman Training School for Boys.

Source: Lyman School for Boys, 100 Years of Progress in the Making of Men, Westborough, Mass., 1947 (?).

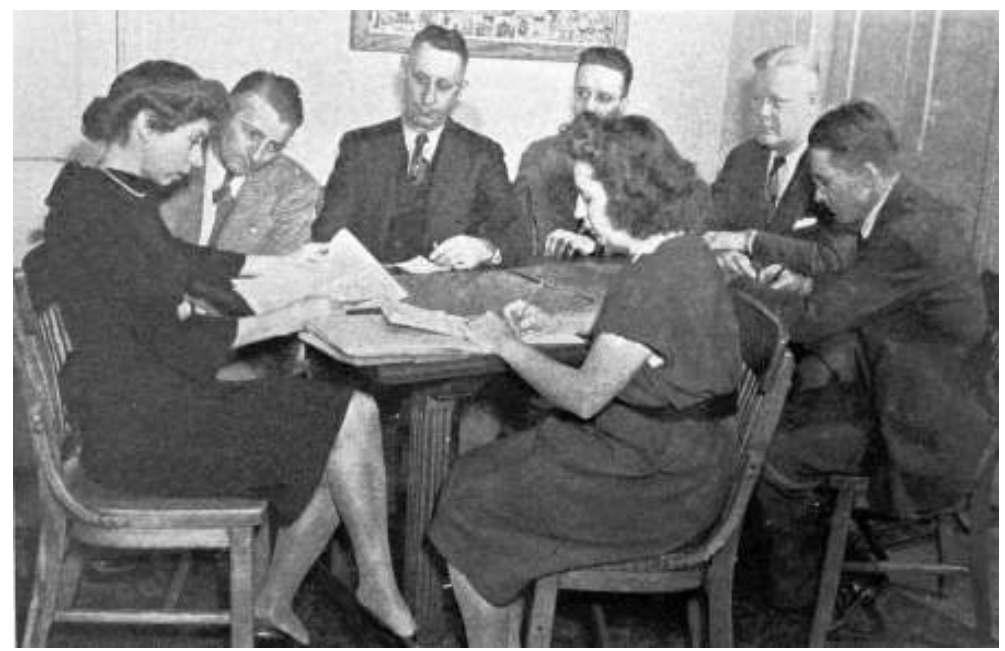

Illustration 5. « The right boy in the right place » (le garcon qu'il faut à l'endroit où il faut) : une réunion de travail à la Lyman Training School for Boys (années 1940).

Source: Lyman School for Boys, 100 Years of Progress in the Making of Men, Westborough, Mass., 1947 (?). 
47. Althusser Louis, "Idéologie et appareils idéologiques d'État (Notes pour une recherche) ", avril 1970, Positions, Paris,

Éditions sociales, 1982,

$$
\text { p. } 133 .
$$

48. Horn Margo, Before It's Too Late..., op. cit.

49. JONES Kathleen W., Taming the Troublesome Child..., op. cit. 50. Ibid.

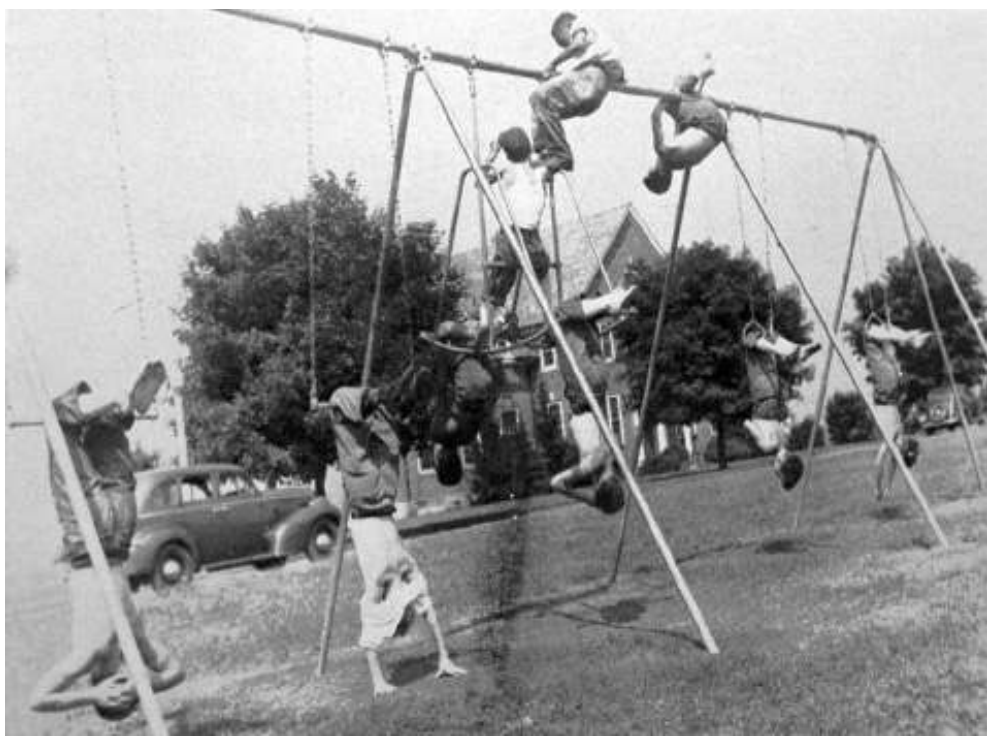

Illustration 6. « Profit from fun » (les bienfaits de l'amusement) à la Lyman Training School for Boys (années 1940).

Source: Lyman School for Boys, 100 years of Progress in the Making of Men, Westborough, Mass., 1947 (?).

L'orientation de plus en plus idéologique de l'appareil psychiatrique, et de l'appareil judiciaire auquel il se relie ici, vise à faire " marcher tout seuls ${ }^{47}$ " les sujets sans qu'aucune forme de répression soit nécessaire, signant là une évolution majeure dans l'économie du pouvoir. Celui-ci s'exerce de manière moins violente, mais plus insidieuse et plus continue, jouant sur la persuasion et touchant de plus en plus de monde suivant la logique du net-widening. La guidance infantile va ainsi étendre son influence bien au-delà des appareils d'État, eux-mêmes renforcés par le Welfare State. Elle s'insère dans toute une série de micro-pouvoirs agissant au quotidien, en premier lieu dans la famille, comme l'ont montré Margo Horn $^{48}$ ou Kathleen W. Jones ${ }^{49}$, où le comportement de la mère est particulièrement scruté. Le blâme ne s'adresse pas seulement à la « mauvaise " mère issue des milieux populaires, plus spécifiquement ouvriers, urbains et immigrés, ciblée depuis longtemps pour ses mœurs ou son travail qui l'éloigne du foyer et des soins à donner aux enfants. Toutes les mères sont dorénavant suspectes, y compris celles des classes moyennes, voire supérieures, auxquelles on reproche leur excès d'amour ${ }^{50}$. En ce sens, le docteur Spock est un héritier du docteur Healy, comme 
les psychiatres et psychologues qui feront un usage extensif du DSM et de ses dérivés, recherchant le développement optimal de l'individu, et accroissant de cette manière leur domaine d'expertise ${ }^{51}$.

Les idées et pratiques du docteur Healy en matière de psychiatrie infantile et de délinquance juvénile ont profondément marqué, aux niveaux américain et international, le domaine de l'" enfance inadaptée » - le concept d'inadaptation, qu'il participe à créer, étendant le champ d'intervention de sa spécialité au-delà du noyau dur de la maladie. Il invente la technique de guidance en s'inspirant des différentes sciences du psychisme qu'il tente de synthétiser et de faire progresser vers un idéal de compréhension du sujet à des fins de normalisation douce. La guidance se décline sous de multiples formes. Modulation de la psychiatrie infantile, elle ajuste son traitement aux situations par un dialogue de type psychothérapeutique qu'elle essaye de ne jamais rompre. Elle évite de retirer le jeune de sa famille, qu'elle participe également à normaliser et à contrôler à travers la prise en charge, essentiellement individuelle, de ses membres.

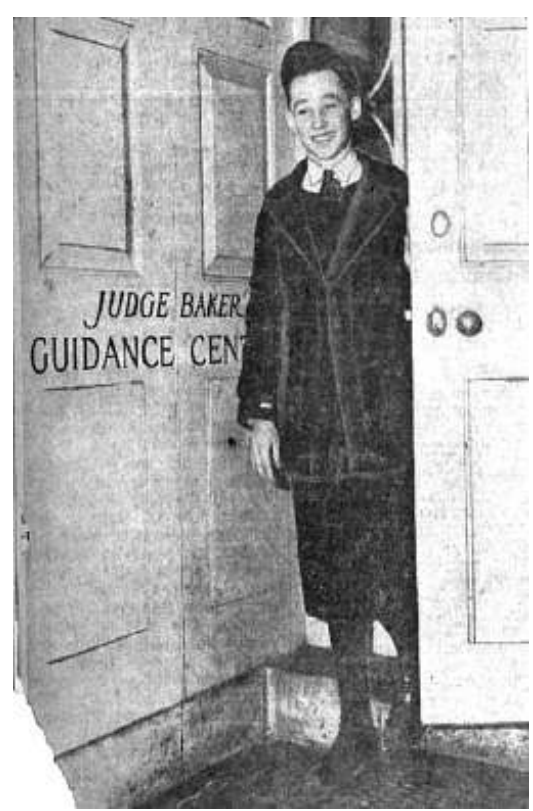

Illustration 7. Un enfant à l'entrée du Judge Baker Guidance Center de Boston.

Source: Photographie sans date (après 1933, quand la Judge Baker Foundation changea de nom pour devenir le Judge Baker Guidance Center). Copyright @ 2016 Judge Baker Children's Center. Used with permission.
51. LUNBECK Elizabeth, The Psychiatric Persuasion: Knowledge, Gender, and Power in Modern America, Princeton, N. J., Princeton University Press, 1994. 
52. Voir l'exemple de Richard donné par JonES Kathleen W., " "Mother Made Me Do It"... », op. cit.

53. Deleuze Gilles, « Postscriptum... », op. cit ., p. 244 et p. 247.
À l'entrée du Judge Baker Guidance Center, un adolescent prend la pose, souriant (ill. 7). Qui est-il? Un jeune délinquant auquel la justice a imposé de suivre une psychothérapie durant sa probation et qui s'en trouve amélioré? Un cancre, qu'une mère anxieuse a amené en consultation, découvrant son potentiel intellectuel grâce à des tests de $\mathrm{Q}^{52}$ et ne devinant pas encore qu'il devra se montrer à la hauteur de ces résultats? On ne sait pas, comme on ne sait pas vraiment s'il se trouve en dedans ou en dehors du centre de guidance. La correction, et même l'amélioration volontaire de soi qu'encouragent les sciences du psychisme dans leur nouvelle version, ne connaît plus les limites de l'institution. Si Gilles Deleuze comparait à une taupe la société disciplinaire, fondée sur le modèle analogique de la prison, il utilisait la métaphore du serpent pour les nouvelles sociétés de contrôle, car «l'homme du contrôle est plutôt ondulatoire, mis en orbite, sur faisceau continu " - comme l'homme des flux du schéma de Healy et Bronner. Appelant à développer les recherches sur les régimes de domination contemporains, sans se leurrer sur les bénéfices immédiats d'un progrès apparent, il prévenait: «Les anneaux d'un serpent sont encore plus compliqués que les trous d'une taupinière ${ }^{53}$." 